\title{
Influence of Frailty on Outcome in Older Patients Undergoing Non-Cardiac Surgery - A Systematic Review and Meta-Analysis
}

\author{
Elke K.M. Tjeertes ${ }^{1}$, Joris M.K. van Fessem ${ }^{1}$, Francesco U.S. Mattace-Raso², Anton G.M. \\ Hoofwijk $^{3}$, Robert Jan Stolker ${ }^{1}$, Sanne E. Hoeks ${ }^{1^{*}}$ \\ ${ }^{1}$ Department of Anesthesiology, Erasmus MC University Medical Center, Rotterdam, the Netherlands \\ ${ }^{2}$ Department of Internal Medicine, Division of Geriatric Medicine, Erasmus MC University Medical Center, \\ Rotterdam, the Netherlands \\ ${ }^{3}$ Department of Surgery, Zuyderland Medical Center, Geleen, the Netherlands
}

[Received August 7, 2019; Revised October 23, 2019; Accepted October 24, 2019]

\begin{abstract}
Frailty is increasingly recognized as a better predictor of adverse postoperative events than chronological age. The objective of this review was to systematically evaluate the effect of frailty on postoperative morbidity and mortality. Studies were included if patients underwent non-cardiac surgery and if frailty was measured by a validated instrument using physical, cognitive and functional domains. A systematic search was performed using EMBASE, MEDLINE, Web of Science, CENTRAL and PubMed from 1990 - 2017. Methodological quality was assessed using an assessment tool for prognosis studies. Outcomes were 30-day mortality and complications, one-year mortality, postoperative delirium and discharge location. Meta-analyses using random effect models were performed and presented as pooled risk ratios with confidence intervals and prediction intervals. We included 56 studies involving 1.106.653 patients. Eleven frailty assessment tools were used. Frailty increases risk of 30-day mortality (31 studies, 673.387 patients, risk ratio 3.71 [95\% CI 2.89-4.77] (PI 1.38-9.97; I2=95\%) and 30-day complications (37 studies, 627.991 patients, RR 2.39 [95\% CI 2.02-2.83). Risk of 1-year mortality was threefold higher (six studies, 341.769 patients, RR 3.40 [95\% CI 2.42-4.77]). Four studies $(\mathrm{N}=438)$ reported on postoperative delirium. Meta-analysis showed a significant increased risk $(\mathrm{RR} 2.13$ [95\% CI 1.23-3.67). Finally, frail patients had a higher risk of institutionalization (10 studies, RR 2.30 [95\% CI 1.81 2.92]). Frailty is strongly associated with risk of postoperative complications, delirium, institutionalization and mortality. Preoperative assessment of frailty can be used as a tool for patients and doctors to decide who benefits from surgery and who doesn't.
\end{abstract}

Key words: frailty, surgery, outcome, older patients, non-cardiac surgery

Life expectancy has increased with the focus on the quality of added life-years [1]. This prolonged life expectancy has created an increased demand for surgical care of the elderly $[2,3]$.

Several studies have described age as an independent risk factor for postoperative morbidity and mortality in both cardiac and non-cardiac surgery [4-7]. Advantages in operative techniques and perioperative management seem to improve outcome and multiple studies have even demonstrated an improved quality of life and enhancement of functional status after cardiac surgery in octogenarians [8-10]. Despite these improvements in perioperative care, postoperative adverse effects still remain more common in older patients when compared to

*Correspondence should be addressed to: Dr. Sanne E. Hoeks, Department of Anaesthesiology, Erasmus MC University Medical Center, Room NA-1720, 3000 CA Rotterdam, the Netherlands. E-mail: s.hoeks@erasmusmc.nl.

Copyright: () 2019 Tjeertes EKM, et al. This is an open-access article distributed under the terms of the Creative Commons Attribution License, which permits unrestricted use, distribution, and reproduction in any medium, provided the original author and source are credited. 
the younger ones $[5,11]$. Adequate risk assessment integrates surgical factors and factors that describe the biological status of the patient, rather than age alone, as age per se seems to be responsible for only a small increase in adverse events [3, 12].

Recently the concept of frailty has come into view [2]. Frailty can be defined as a clinically recognizable state of increased vulnerability resulting from agingassociated lack of physiological reserve and decline in function across multiple physiologic systems [13]. Focus on and optimization of frail patients can contribute to a reduced postoperative morbidity and thereby to better outcome in the older surgical population [2]. Globally, the World Health Organisation has recently developed recommendations on integrated care for older patients in order to maintain their physical and cognitive functions [14].

In order to adequately inform our patients of significant perioperative risks, additional information on frailty as a risk factor influencing postoperative outcome is essential. During the preoperative assessment, this information can guide the clinician in shared decision making on whether the older patient benefits from surgery or not. The aim of this study was to evaluate the predictive role of frailty on postoperative outcomes after non-cardiac surgery by conducting a systematic review and metaanalysis of literature.

\section{METHODS}

\section{Search Strategy}

A search of literature was performed and reported according to the Preferred Reporting Items for Systematic Reviews and Meta-analyses (PRISMA) statement and MOOSE criteria [15]. The objective was to find all studies on frail patients undergoing non-cardiac surgery, correlating their age and its subsequent risk factors to postoperative morbidity and mortality. The systematic Internet based search was performed using EMBASE, MEDLINE, Web of Science, Cochrane Central Register of Controlled Trials (CENTRAL) and PubMed. Full electronic searches can be found in Supplementary Table. 1. In addition, we screened the reference section of all articles included in this review. The search was limited to original articles, human subjects and articles published from January 1990 - December 2017.

\section{Publication selection}

Two reviewers independently (EKMT and JMKvF) screened potentially relevant articles from the initial search, first by title and abstract and later on by full text. Any disagreements between the two reviewers were resolved by discussion and consensus with a third reviewer (SH). Studies were found eligible for inclusion if their subjects underwent non-cardiac surgery and if frailty was measured by a frailty instrument using at least physical, cognitive and functional domains. Also, the relationship between frailty and primary outcomes of 30day mortality, or 30-day complications should be evaluated, with stratification of the outcome (frail versus non-frail). Studies were excluded if they were review articles, case reports, editorials or comments, or if full text was not available. Duplicate articles were removed during the initial search.

\section{Data Extraction}

The following data were gathered from eligible publications: publication date, study design, sample size, type of surgery, proportion of females, mean age, the frailty score and outcome. Outcome was measured by the following adverse events: 30-day mortality, 30-day complications, one-year mortality, manifestation of postoperative delirium (POD) and discharge to a specialized facility. 30-day complications are generally defined as suggested by the Clavien-Dindo classification system[16]; otherwise the authors should have predefined this outcome. Postoperative delirium was defined as a temporary state of confusion and diagnosis made with validated delirium screening tools or by a geriatric expert team [17]. Discharge destination was defined as "home", or "not able to return home". Furthermore, surgical procedures were categorised according to the ESC/ESA Guidelines [18] and divided into low-, intermediate- and high-risk procedures. Occasionally, the surgical risk category was documented as "mixed surgical population". A subanalysis per surgery type was performed to better understand the effect of frailty according to the surgical risk category. Where absolute data were not presented in table or text and authors could not be reached, when possible, data were extracted from figures using WebPlotDigitizer (version, 2.6.8).

\section{Assessment of quality and possible biases}

Two reviewers performed assessment of quality. In case of disagreement a third reviewer was consulted. The quality assessment tool for prognosis studies as proposed by Hayden et al. was used for the appraisal of all included studies [19]. This tool focuses on six areas of potential bias; first study participation (i.e. the study sample represents the population of interest on key characteristics), second study attrition (i.e. whether the study was able to obtain a complete follow up), third prognostic factor measurement (i.e. a clear definition or description of the prognostic factor measured is 
provided), fourth outcome measurement (i.e. a clear definition of the outcome of interest), fifth confounding measurement and account (i.e. important potential confounders are appropriately accounted for) and sixth analysis (i.e. the statistical analysis is appropriate for the design of the study). After the evaluation of these six areas of potential bias, all studies were subsequently divided According to the Quality in Prognosis Study Tool into good (11 or 12 points), fair (9 or 10 points) and poor $(<9$ points) quality.

\section{Statistical methods}

Numerical values reported by the studies were used for analysis. In some cases, further calculation was required for ascertaining outcomes. In the studies using the modified frailty index $(\mathrm{mFI})$ patients were categorized into two groups: "not frail" $(\mathrm{mFI}<0.27)$, or "frail" (mFI $\geq 0.27$ ). The decision to divide patients into those categories was based on thresholds most commonly used to indicate the presence of frailty and was made before analysis. In the remaining studies, using ten different frailty instruments, outcome was also dichotomized according to predefined criteria as "not frail" or "frail". Random effects models for meta-analysis were used because of the large expected heterogeneity in determinant and other study characteristics. The primary outcome measures 30-day mortality and 30-day complications were stratified by frailty score. Furthermore, a subanalysis per surgery type was performed to better understand the effect of frailty according to the surgical risk category. Effect estimates are presented as pooled risk ratios (RR) with 95\% confidence intervals (CI's). Robust meta-analytic conclusions of prognosis studies will be more appropriately signaled when prediction intervals are provided [20]. Thus, to further account for between-study heterogeneity, 95\% prediction interval (PI) were also estimated, which evaluates the uncertainty of the effect that would be expected in a new study addressing the same association [21]. $\mathrm{I}^{2}$ statistic was calculated, which is the percentage of variation across studies due to heterogeneity rather than random error. Since all reported outcomes were adverse events, a positive relative risk indicates that frailty is associated with worse patient outcome. A meta-regression analysis was carried out to assess the influence of the patient's mean age (using mean or median age of the study populations as a proxy) on 30day mortality. Finally, an additional sensitivity analysis was performed (excluding studies using ACS-NSQIP database) to circumvent the issue of possible duplicate cases and demonstrate the effect of frailty on postoperative outcome.
Data gathering and data analysis was performed using Excel (version 14.7.2) and Rstudio (version 1.1.463) respectively.

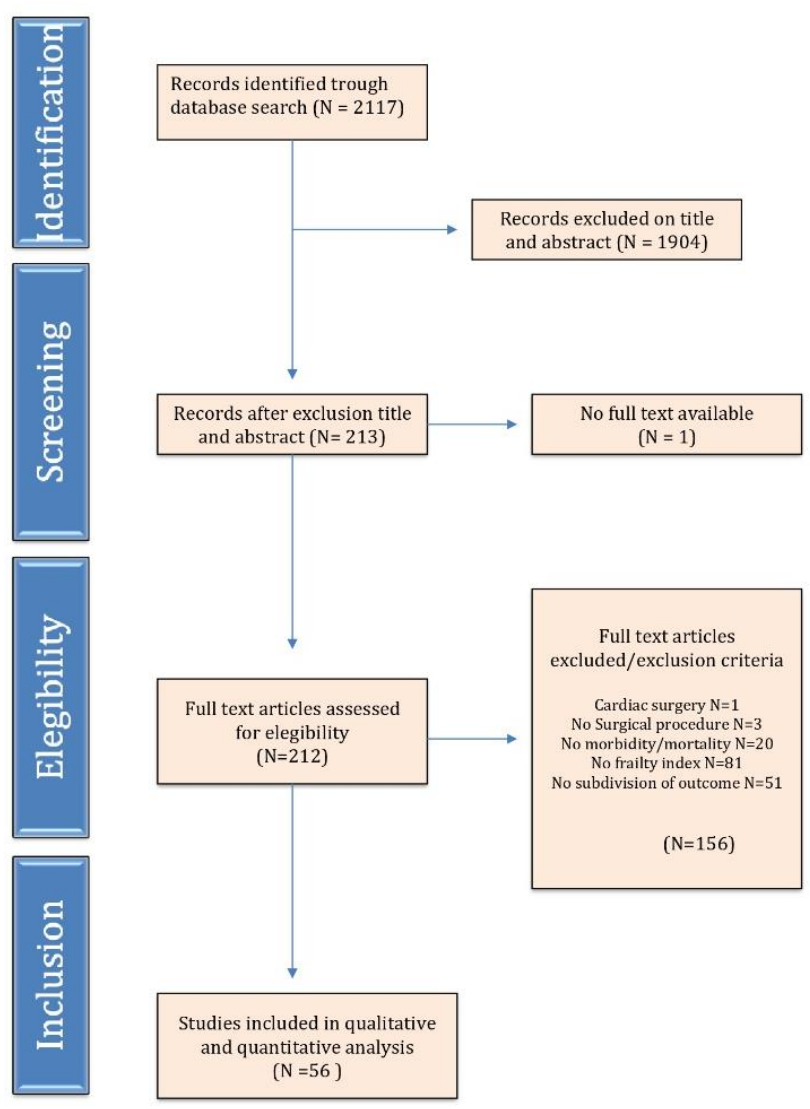

Figure 1. PRISMA flowchart for study selection. This flowchart depicts the flow of information trough different phases of the systematic research.

\section{RESULTS}

Initial literature search identified 2117 manuscripts as potentially relevant. Of these, 1904 were excluded due to unrelated research questions or study type. Full text was not available in one study; therefore 212 full text articles were thoroughly screened for eligibility. A total of 56 studies were found suitable for this systematic review. Figure 1 shows the search strategy flow chart.

\section{Frailty assessment tools}

A total of eleven different frailty assessment tools were used. The majority of studies (twenty-four) used the Modified Frailty Index (mFI), created by Saxton and Velanovich [22]. The mFI consists of eleven variables present in the Canadian Study on Health and Aging Frailty Index, as well as in the American College of Surgeons National Surgical Quality Improvement Program (ACS 
NSQIP) dataset [23, 24]. Variations on the Fried Frailty Criteria [25] were used in eleven studies, where frailty was defined by identifying unintentional weight loss, exhaustion, low energy expenditure, low grip strength and slow walking speed. Frailty assessment tools were often based on comprehensive geriatric assessments, which can be derived from questionnaires or patient files, including the Frailty Index and the Groningen Frailty Indicator. Supplementary Fig. 2 provides a detailed description of all frailty assessment tools used in this review.

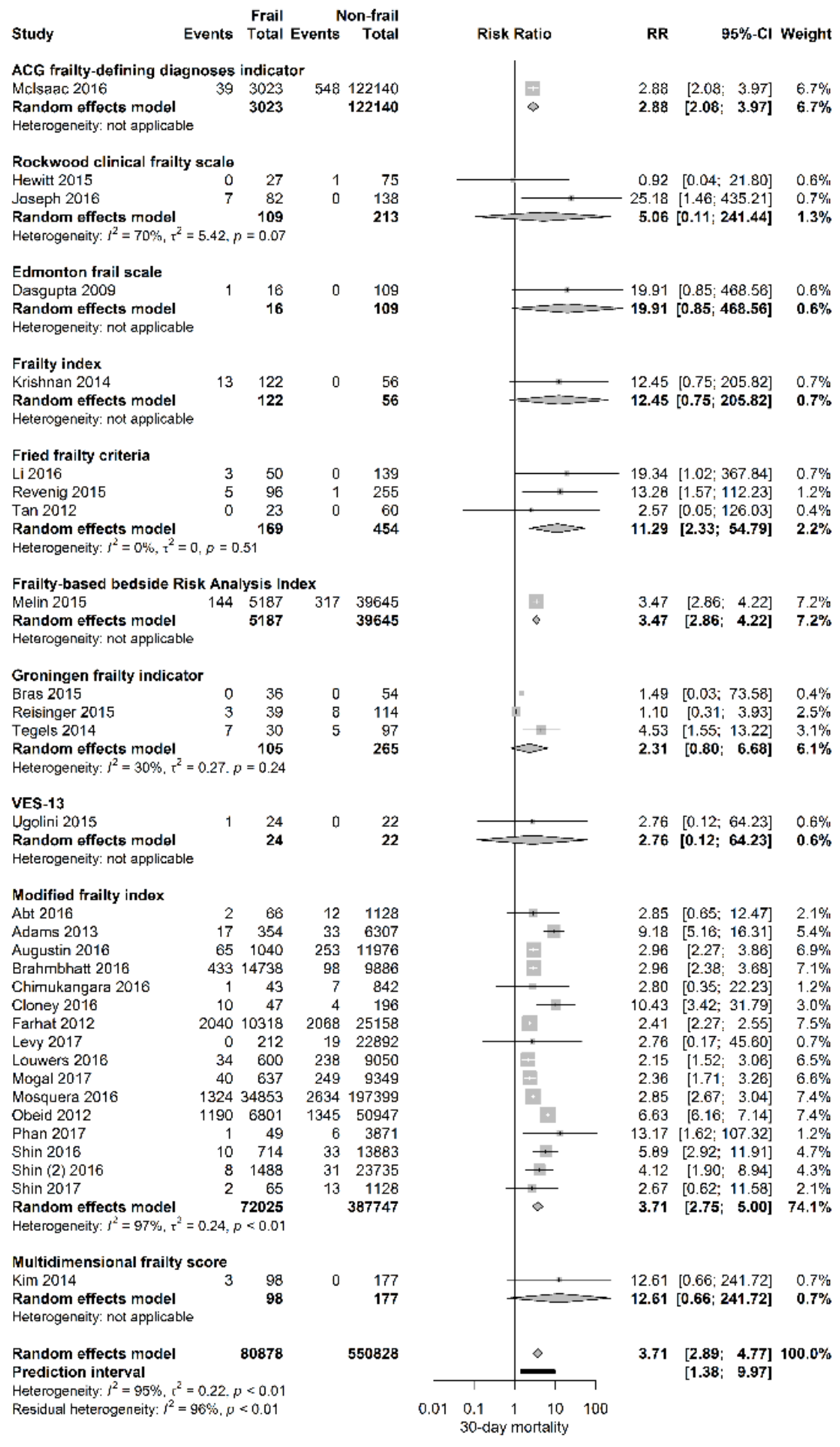

Figure 2. Forest plot 30-day mortality per frailty score. The number of events (deaths) and the total number of patients are shown for both frail and non-frail patients, stratified per frailty assessment tool. 


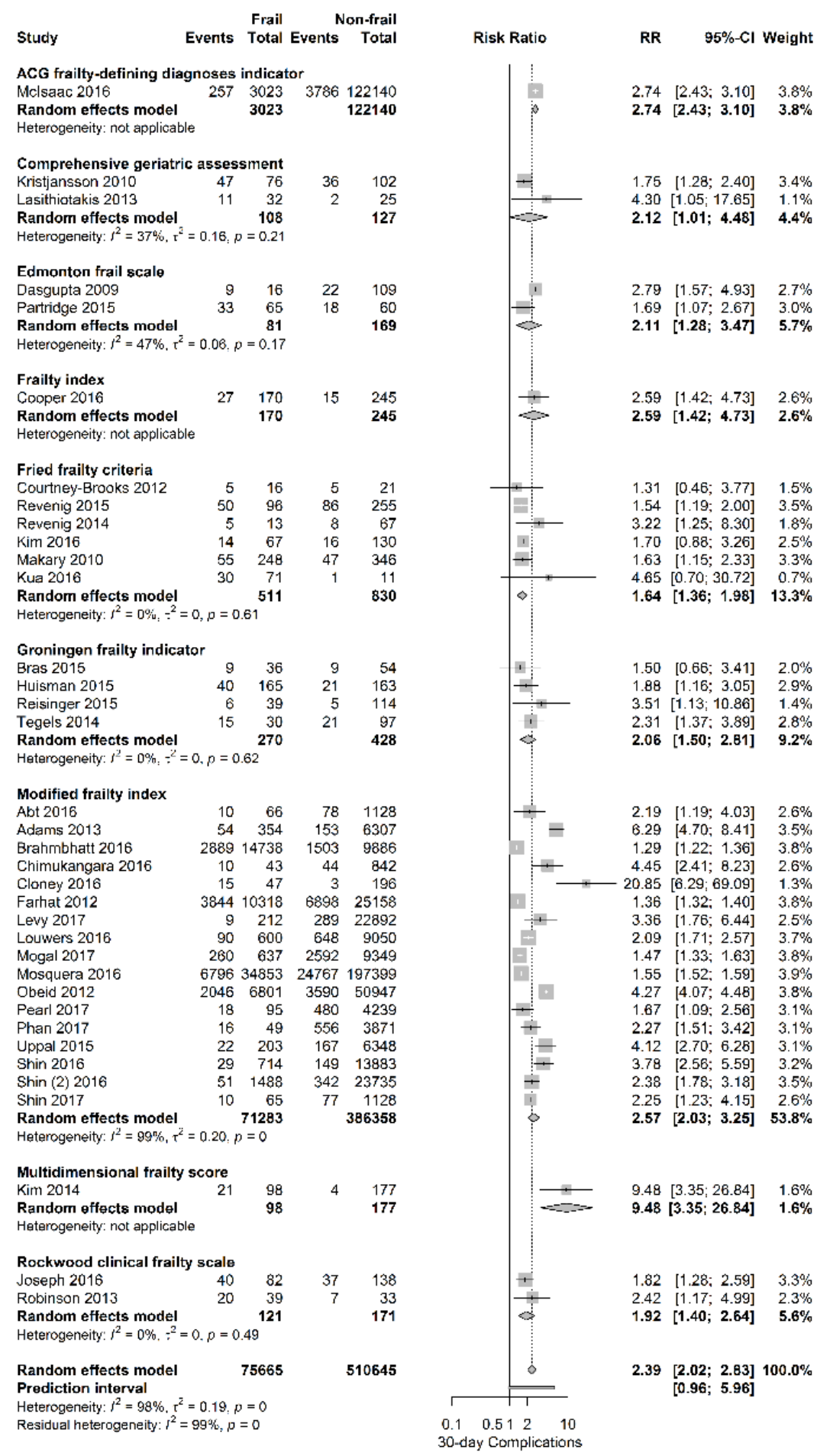

Figure 3. Forest plot postoperative complications per frailty score. The number of events (complications) and the total number of patients are shown for both frail and nonfrail patients, stratified per frailty assessment tool.

\section{Quality assessment}

The quality assessment of the included studies is provided in Supplementary Fig. 3 and table 1 provides a summary of our appraisal. Study participation was adequately described in 37 studies. The study attrition - referring to the response rate and attempts to collect information on patients who were lost to follow up - was adequately defined in 40 studies. Prognostic factors were clearly defined or described in most studies (86\%). Ninety-one 
percent of studies provided a clear definition of the outcome of interest. When summarizing, $95 \%$ of all studies included were of at least fair quality, with more than half assessed as good quality.

Table 1. Study demographics and method of determining frailty.

\begin{tabular}{|c|c|c|c|c|c|c|c|c|}
\hline Author & $\mathbf{N}$ & Setting & Period & Design & Type of surgery & Frailty score & $\begin{array}{l}\text { Definition of } \\
\text { complication }\end{array}$ & Quality \\
\hline $\mathrm{Abt}$ & 1193 & $\begin{array}{l}\text { Multicenter cohort } \\
\text { study (NSQIP) }\end{array}$ & $\begin{array}{l}2006- \\
2013\end{array}$ & Prospective & $\begin{array}{l}\text { Head and neck cancer } \\
\text { surgery }\end{array}$ & $\begin{array}{l}\text { Modified frailty } \\
\text { index }\end{array}$ & $\mathrm{CD} 4$ & Good \\
\hline Adams & 6727 & $\begin{array}{l}\text { Multicenter cohort } \\
\text { study (NSQIP) }\end{array}$ & $\begin{array}{l}2005- \\
2010\end{array}$ & Prospective & $\begin{array}{l}\text { Head and neck cancer } \\
\text { surgery }\end{array}$ & $\begin{array}{l}\text { Modified frailty } \\
\text { index }\end{array}$ & $\mathrm{CD} 4$ or 5 & Good \\
\hline Arya & 23027 & $\begin{array}{l}\text { Multicenter cohort } \\
\text { study (NSQIP) }\end{array}$ & $\begin{array}{l}2005- \\
2012\end{array}$ & Prospective & $\begin{array}{l}\text { Vascular surgery (Open } \\
\text { or EVAR) }\end{array}$ & $\begin{array}{l}\text { Modified frailty } \\
\text { index }\end{array}$ & $\mathrm{CD} 4$ & Good \\
\hline Augustin & 13020 & $\begin{array}{l}\text { Multicenter cohort } \\
\text { study (NSQIP) }\end{array}$ & $\begin{array}{l}2005- \\
2010\end{array}$ & Prospective & Pancreatic resections & $\begin{array}{l}\text { Modified frailty } \\
\text { index }\end{array}$ & $\mathrm{CD} 4$ & Good \\
\hline Brahmbhatt & 24645 & $\begin{array}{l}\text { Multicenter cohort } \\
\text { study (NSQIP) }\end{array}$ & $\begin{array}{l}2005- \\
2012\end{array}$ & Prospective & $\begin{array}{l}\text { Infrainguinal vascular } \\
\text { surgery }\end{array}$ & $\begin{array}{l}\text { Modified frailty } \\
\text { index }\end{array}$ & CD 4 & Good \\
\hline Bras & 90 & $\begin{array}{l}\text { Single-center } \\
\text { cohort study }\end{array}$ & $\begin{array}{l}2008- \\
2013\end{array}$ & Retrospective & $\begin{array}{l}\text { Surgery for head and neck } \\
\text { cancer }\end{array}$ & $\begin{array}{l}\text { Groningen frailty } \\
\text { indicator }\end{array}$ & $\mathrm{CD} \geq 2$ & Fair \\
\hline Chappidi & 2679 & $\begin{array}{l}\text { Multicenter cohort } \\
\text { study (NSQIP) }\end{array}$ & $\begin{array}{l}2011- \\
2013\end{array}$ & Prospective & Radical cystectomy & $\begin{array}{l}\text { Modified frailty } \\
\text { index }\end{array}$ & CD 4 or 5 & Good \\
\hline Chimukangara & 885 & $\begin{array}{l}\text { Multicenter cohort } \\
\text { study (NSQIP) }\end{array}$ & $\begin{array}{l}2011- \\
2013\end{array}$ & Prospective & $\begin{array}{l}\text { Paraesofageal hernia } \\
\text { repair }\end{array}$ & $\begin{array}{l}\text { Modified frailty } \\
\text { index }\end{array}$ & $\mathrm{CD} \geq 3$ & Fair \\
\hline Cloney & 243 & $\begin{array}{l}\text { Multicenter cohort } \\
\text { study (NSQIP) }\end{array}$ & $\begin{array}{l}2000- \\
2012\end{array}$ & Prospective & Glioblastoma surgery & $\begin{array}{l}\text { Modified frailty } \\
\text { index }\end{array}$ & $\begin{array}{l}\text { Complications } \\
\text { (Glioma } \\
\text { Outcomes } \\
\text { Project } \\
\text { System) }\end{array}$ & Fair \\
\hline Cooper & 415 & $\begin{array}{l}\text { Multicenter cohort } \\
\text { study }\end{array}$ & $\begin{array}{l}2010- \\
2013\end{array}$ & Prospective & $\begin{array}{l}\text { General and orthopedic } \\
\text { surgery }\end{array}$ & $\begin{array}{l}\text { Frailty } \\
\text { phenotype; } \\
\text { frailty index }\end{array}$ & $\begin{array}{l}\text { Major } \\
\text { complications }\end{array}$ & Fair \\
\hline $\begin{array}{l}\text { Courtney- } \\
\text { Brooks }\end{array}$ & 37 & $\begin{array}{l}\text { Single-center } \\
\text { cohort study }\end{array}$ & 2011 & Prospective & $\begin{array}{l}\text { Surgery for gynecologic } \\
\text { cancer }\end{array}$ & $\begin{array}{l}\text { Fried frailty } \\
\text { criteria }\end{array}$ & $\begin{array}{l}\text { Surgical } \\
\text { complications } \\
\text { (NSQIP) }\end{array}$ & Fair \\
\hline Dale & 76 & $\begin{array}{l}\text { Single-center } \\
\text { cohort study }\end{array}$ & $\begin{array}{l}2007- \\
2011\end{array}$ & Prospective & Pancreaticoduodenectomy & $\begin{array}{l}4 \text { (of 5) } \\
\text { components of } \\
\text { Fried frailty } \\
\text { criteria; VES-13 }\end{array}$ & $\mathrm{CD} \geq 3$ & Fair \\
\hline Dasgupta & 125 & $\begin{array}{l}\text { Single-center } \\
\text { cohort study }\end{array}$ & $\begin{array}{l}2002- \\
2003\end{array}$ & Prospective & $\begin{array}{l}\text { Elective noncardiac } \\
\text { surgery }(82 \%) \\
\text { orthopedic) }\end{array}$ & $\begin{array}{l}\text { Edmonton frail } \\
\text { scale }\end{array}$ & $\begin{array}{l}\text { Cardiac - / } \\
\text { pulmonary } \\
\text { comlications, } \\
\text { POD }\end{array}$ & Fair \\
\hline Farhat & 35334 & $\begin{array}{l}\text { Multicenter cohort } \\
\text { study (NSQIP) }\end{array}$ & $\begin{array}{l}2005- \\
2009\end{array}$ & Prospective & $\begin{array}{l}\text { Emergency general } \\
\text { surgery }\end{array}$ & $\begin{array}{l}\text { Modified frailty } \\
\text { index }\end{array}$ & $\begin{array}{l}\text { Any } \\
\text { complication } \\
\text { (not } \\
\text { mortality) }\end{array}$ & Fair \\
\hline Flexman & 52671 & $\begin{array}{l}\text { Multicenter cohort } \\
\text { study (NSQIP) }\end{array}$ & $\begin{array}{l}2006- \\
2012\end{array}$ & Prospective & Spine surgery & $\begin{array}{l}\text { Modified frailty } \\
\text { index }\end{array}$ & $\begin{array}{l}\text { Major } \\
\text { complications }\end{array}$ & Good \\
\hline Hewitt & 102 & $\begin{array}{l}\text { Multicenter cohort } \\
\text { study }\end{array}$ & 2013 & Prospective & $\begin{array}{l}\text { Emergency general } \\
\text { surgery }\end{array}$ & $\begin{array}{l}\text { Rockwood } \\
\text { clinical frailty } \\
\text { scale }\end{array}$ & Not reported & Fair \\
\hline Huisman & 328 & $\begin{array}{l}\text { Multicenter cohort } \\
\text { study }\end{array}$ & $\begin{array}{l}2008- \\
2012\end{array}$ & Prospective & Surgery for solid tumors & $\begin{array}{l}\text { Groningen frailty } \\
\text { indicator; VES- } \\
13\end{array}$ & $\mathrm{CD} \geq 3$ & Good \\
\hline Joseph & 220 & $\begin{array}{l}\text { Single-center } \\
\text { cohort study }\end{array}$ & $\begin{array}{l}2012- \\
2014\end{array}$ & Prospective & $\begin{array}{l}\text { Emergency general } \\
\text { surgery }\end{array}$ & $\begin{array}{l}\text { Rockwood } \\
\text { clinical frailty } \\
\text { scale }\end{array}$ & $\begin{array}{l}\text { Surgical } \\
\text { complications } \\
\text { (NSQIP) }\end{array}$ & Fair \\
\hline Kenig & 184 & $\begin{array}{l}\text { Single-center } \\
\text { cohort study }\end{array}$ & $\begin{array}{l}2013- \\
2014\end{array}$ & Prospective & $\begin{array}{l}\text { Emergency abdominal } \\
\text { surgery }\end{array}$ & $\begin{array}{l}\text { VES-13, GFI; } \\
\text { Rockwood; } \\
\text { Balducci; TRST; } \\
\text { Geriatric-8 }\end{array}$ & $\begin{array}{l}\text { Any } \\
\text { complication } \\
\text { (CD) }\end{array}$ & Fair \\
\hline Kim & 197 & $\begin{array}{l}\text { Single-center } \\
\text { cohort study }\end{array}$ & $\begin{array}{l}2012- \\
2014\end{array}$ & Prospective & $\begin{array}{l}\text { Elective noncardiac } \\
\text { surgery }\end{array}$ & $\begin{array}{l}\text { Fried frailty } \\
\text { criteria }\end{array}$ & $\begin{array}{l}\text { Surgical } \\
\text { complications } \\
\text { (NSQIP) }\end{array}$ & Good \\
\hline Kim & 275 & $\begin{array}{l}\text { Single-center } \\
\text { cohort study }\end{array}$ & $\begin{array}{l}2011- \\
2012\end{array}$ & Prospective & $\begin{array}{l}\text { Elective intermediate-risk } \\
\text { or high-risk surgery }\end{array}$ & $\begin{array}{l}\text { Multidimensional } \\
\text { frailty score }\end{array}$ & $\begin{array}{l}\text { Surgical } \\
\text { complications } \\
\text { (NSQIP) }\end{array}$ & Good \\
\hline Krishnan & 178 & $\begin{array}{l}\text { Single-center } \\
\text { cohort study }\end{array}$ & 2011 & Prospective & $\begin{array}{l}\text { Low trauma hip fracture } \\
\text { surgery }\end{array}$ & Frailty index & Not reported & Poor \\
\hline
\end{tabular}




\begin{tabular}{|c|c|c|c|c|c|c|c|c|}
\hline Kristjansson & 178 & $\begin{array}{l}\text { Multicenter cohort } \\
\text { study }\end{array}$ & $\begin{array}{l}2008- \\
2011\end{array}$ & Prospective & $\begin{array}{l}\text { Elective surgery for } \\
\text { colorectal cancer }\end{array}$ & $\begin{array}{l}\text { Comprehensive } \\
\text { geriatric } \\
\text { assessment }\end{array}$ & $\mathrm{CD} \geq 2$ & Good \\
\hline Kua & 82 & $\begin{array}{l}\text { Single-center } \\
\text { cohort study }\end{array}$ & 2013 & Prospective & Hip fracture surgery & $\begin{array}{l}\text { Edmonton frail } \\
\text { scale; (modified) } \\
\text { Fried frailty } \\
\text { criteria }\end{array}$ & $\begin{array}{l}\text { Any } \\
\text { complication }\end{array}$ & Fair \\
\hline Lascano & 41681 & $\begin{array}{l}\text { Multicenter cohort } \\
\text { study (NSQIP) }\end{array}$ & $\begin{array}{l}2005- \\
2013\end{array}$ & Prospective & $\begin{array}{l}\text { Surgery for urologic } \\
\text { cancer }\end{array}$ & $\begin{array}{l}\text { Modified frailty } \\
\text { index }\end{array}$ & $\mathrm{CD} 4$ & Good \\
\hline Lasithiotakis & 57 & $\begin{array}{l}\text { Single-center } \\
\text { cohort study }\end{array}$ & $\begin{array}{l}2008- \\
2011\end{array}$ & Prospective & $\begin{array}{l}\text { Elective laparoscopic } \\
\text { cholecystectomy }\end{array}$ & $\begin{array}{l}\text { Comprehensive } \\
\text { geriatric } \\
\text { assessment }\end{array}$ & $\begin{array}{l}\text { Any } \\
\text { complication }\end{array}$ & Poor \\
\hline Leung & 63 & $\begin{array}{l}\text { Single-center } \\
\text { cohort study }\end{array}$ & 2007 & Prospective & Noncardiac surgery & $\begin{array}{l}\text { Fried frailty } \\
\text { criteria }\end{array}$ & Not reported & Fair \\
\hline Levy & 23104 & $\begin{array}{l}\text { Multicenter cohort } \\
\text { study (NSQIP) }\end{array}$ & $\begin{array}{l}2008 \text { to } \\
2014\end{array}$ & Prospective & $\begin{array}{l}\text { Robot-assisted radical } \\
\text { prostatectomy }\end{array}$ & $\begin{array}{l}\text { Modified frailty } \\
\text { index }\end{array}$ & $\mathrm{CD} 4$ & Good \\
\hline $\mathrm{Li}$ & 189 & $\begin{array}{l}\text { Single-center } \\
\text { cohort study }\end{array}$ & $\begin{array}{l}\text { Not } \\
\text { reported }\end{array}$ & Prospective & $\begin{array}{l}\text { Major intra-abdominal } \\
\text { surgery }\end{array}$ & $\begin{array}{l}\text { Fried frailty } \\
\text { criteria }\end{array}$ & $\mathrm{CD}$ & Fair \\
\hline Louwers & 10300 & $\begin{array}{l}\text { Multicenter cohort } \\
\text { study (NSQIP) }\end{array}$ & $\begin{array}{l}2005- \\
2011\end{array}$ & Prospective & Hepatectomy & $\begin{array}{l}\text { Modified frailty } \\
\text { index }\end{array}$ & $\mathrm{CD} 4$ & Good \\
\hline Makary & 594 & $\begin{array}{l}\text { Single-center } \\
\text { cohort study }\end{array}$ & $\begin{array}{l}2005- \\
2006\end{array}$ & Prospective & Elective surgery & $\begin{array}{l}\text { Fried frailty } \\
\text { criteria }\end{array}$ & $\begin{array}{l}\text { Surgical } \\
\text { complications } \\
\text { (NSQIP) }\end{array}$ & Good \\
\hline $\begin{array}{l}\text { McAdams- } \\
\text { DeMarco } \\
\end{array}$ & 537 & $\begin{array}{l}\text { Single-center } \\
\text { cohort study }\end{array}$ & $\begin{array}{l}2008- \\
2013\end{array}$ & Prospective & Kidney transplant surgery & $\begin{array}{l}\text { Fried frailty } \\
\text { criteria }\end{array}$ & Not reported & Fair \\
\hline McIsaac & 202811 & $\begin{array}{l}\text { Single-center } \\
\text { cohort study }\end{array}$ & $\begin{array}{l}2002- \\
2012\end{array}$ & Retrospective & $\begin{array}{l}\text { Major elective noncardiac } \\
\text { surgery }\end{array}$ & $\begin{array}{l}\text { ACG frailty- } \\
\text { defining } \\
\text { diagnoses } \\
\text { indicator }\end{array}$ & Not reported & Good \\
\hline McIsaac & 125163 & $\begin{array}{l}\text { Single-center } \\
\text { cohort study }\end{array}$ & $\begin{array}{l}2003- \\
2012\end{array}$ & Retrospective & Total joint arthroplasty & $\begin{array}{l}\text { ACG frailty- } \\
\text { defining } \\
\text { diagnoses } \\
\text { indicator }\end{array}$ & $\begin{array}{l}\text { ICU- } \\
\text { admission }\end{array}$ & Good \\
\hline Melin & 44832 & $\begin{array}{l}\text { Multicenter cohort } \\
\text { study (NSQIP) }\end{array}$ & $\begin{array}{l}2005- \\
2011\end{array}$ & Prospective & Carotid endarterectomy & $\begin{array}{l}\text { Frailty-based } \\
\text { bedside Risk } \\
\text { Analysis Index }\end{array}$ & Not reported & Fair \\
\hline Mogal & 9986 & $\begin{array}{l}\text { Multicenter cohort } \\
\text { study (NSQIP) }\end{array}$ & $\begin{array}{l}2005- \\
2012\end{array}$ & Prospective & Pancreaticoduodenectomy & $\begin{array}{l}\text { Modified frailty } \\
\text { index }\end{array}$ & CD 3 or 4 & Good \\
\hline Mosquera & 232352 & $\begin{array}{l}\text { Multicenter cohort } \\
\text { study (NSQIP) }\end{array}$ & $\begin{array}{l}2005- \\
2012\end{array}$ & Prospective & elective high-risk surgery & $\begin{array}{l}\text { Modified frailty } \\
\text { index }\end{array}$ & $\begin{array}{l}\text { Major and } \\
\text { minor } \\
\text { complications }\end{array}$ & Fair \\
\hline Neuman & 12979 & $\begin{array}{l}\text { Single-center } \\
\text { cohort study }\end{array}$ & $\begin{array}{l}1992- \\
2005\end{array}$ & Retrospective & $\begin{array}{l}\text { Elective colorectal cancer } \\
\text { surgery }\end{array}$ & $\begin{array}{l}\text { ACG frailty- } \\
\text { defining } \\
\text { diagnoses } \\
\text { indicator }\end{array}$ & $\begin{array}{l}\text { Readmission } \\
\text { within } 30 \\
\text { days }\end{array}$ & Fair \\
\hline Obeid & 58448 & $\begin{array}{l}\text { Multicenter cohort } \\
\text { study (NSQIP) }\end{array}$ & $\begin{array}{l}2005- \\
2009\end{array}$ & Prospective & $\begin{array}{l}\text { Laparoscopic and open } \\
\text { colectomy }\end{array}$ & $\begin{array}{l}\text { Modified frailty } \\
\text { index }\end{array}$ & $\mathrm{CD} 4$ or 5 & Fair \\
\hline Partridge & 125 & $\begin{array}{l}\text { Single-center } \\
\text { cohort study }\end{array}$ & 2011 & Prospective & Arterial vascular surgery & $\begin{array}{l}\text { Edmonton frail } \\
\text { scale }\end{array}$ & $\begin{array}{l}\text { Composite } \\
\text { postoperative } \\
\text { complications }\end{array}$ & Fair \\
\hline Pearl & 4330 & $\begin{array}{l}\text { Multicenter cohort } \\
\text { study (NSQIP) }\end{array}$ & $\begin{array}{l}2011- \\
2014\end{array}$ & Prospective & Radical cystectomy & $\begin{array}{l}\text { Modified frailty } \\
\text { index }\end{array}$ & $\begin{array}{l}\text { Major in- } \\
\text { hospital } \\
\text { complications }\end{array}$ & Good \\
\hline Phan & 3920 & $\begin{array}{l}\text { Multicenter cohort } \\
\text { study (NSQIP) }\end{array}$ & $\begin{array}{l}2010- \\
2014\end{array}$ & Prospective & $\begin{array}{l}\text { Elective anterior lumbar } \\
\text { interbody fusion (ALIF) } \\
\text { surgery }\end{array}$ & $\begin{array}{l}\text { Modified frailty } \\
\text { index }\end{array}$ & $\begin{array}{l}\text { Any } \\
\text { complication }\end{array}$ & Good \\
\hline Reisinger & 159 & $\begin{array}{l}\text { Single-center } \\
\text { cohort study }\end{array}$ & $\begin{array}{l}2010- \\
2012\end{array}$ & Prospective & Colorectal surgery & $\begin{array}{l}\text { Groningen frailty } \\
\text { indicator }\end{array}$ & Sepsis & Good \\
\hline Revenig & 351 & $\begin{array}{l}\text { Single-center } \\
\text { cohort study }\end{array}$ & $\begin{array}{l}\text { Not } \\
\text { reported }\end{array}$ & Prospective & $\begin{array}{l}\text { Major intra-abdominal } \\
\text { surgery }\end{array}$ & $\begin{array}{l}\text { Fried frailty } \\
\text { criteria }\end{array}$ & CD 1-4 & Fair \\
\hline Revenig & 80 & $\begin{array}{l}\text { Single-center } \\
\text { cohort study }\end{array}$ & $\begin{array}{l}\text { Not } \\
\text { reported }\end{array}$ & Prospective & $\begin{array}{l}\text { Intra-abdominal } \\
\text { minimally invasive } \\
\text { surgery }\end{array}$ & $\begin{array}{l}\text { Fried frailty } \\
\text { criteria }\end{array}$ & CD 1-4 & Fair \\
\hline Revenig & 189 & $\begin{array}{l}\text { Single-center } \\
\text { cohort study }\end{array}$ & $\begin{array}{l}\text { Not } \\
\text { reported }\end{array}$ & Prospective & $\begin{array}{l}\text { Major intra-abdominal } \\
\text { surgery }\end{array}$ & $\begin{array}{l}\text { Fried frailty } \\
\text { criteria }\end{array}$ & $\begin{array}{l}\text { Any } \\
\text { complication }\end{array}$ & Good \\
\hline Robinson & 72 & $\begin{array}{l}\text { Single-center } \\
\text { cohort study }\end{array}$ & $\begin{array}{l}2007- \\
2010\end{array}$ & Prospective & Colorectal surgery & $\begin{array}{l}\text { Rockwood } \\
\text { clinical frailty } \\
\text { scale }\end{array}$ & $\begin{array}{l}\text { Any } \\
\text { postoperative } \\
\text { complication } \\
\text { (VASQIP) }\end{array}$ & Fair \\
\hline Shin & $\begin{array}{l}6148 \\
\text { ACDF; }\end{array}$ & $\begin{array}{l}\text { Multicenter cohort } \\
\text { study (NSQIP) }\end{array}$ & $\begin{array}{l}2005- \\
2012\end{array}$ & Prospective & $\begin{array}{l}\text { Cervical spine fusion; } \\
\text { anterior cervical }\end{array}$ & $\begin{array}{l}\text { Modified frailty } \\
\text { index }\end{array}$ & $\mathrm{CD} 4$ & Good \\
\hline
\end{tabular}




\begin{tabular}{|c|c|c|c|c|c|c|c|c|}
\hline & $\begin{array}{l}817 \\
\text { PCF }\end{array}$ & & & & $\begin{array}{l}\text { discectomy and fusion or } \\
\text { posterior cervical fusion }\end{array}$ & & & \\
\hline Shin & $\begin{array}{l}14583 \\
\text { THA; } \\
25223 \\
\text { TKA }\end{array}$ & $\begin{array}{l}\text { Multicenter cohort } \\
\text { study (NSQIP) }\end{array}$ & $\begin{array}{l}2005- \\
2012\end{array}$ & Prospective & $\begin{array}{l}\text { Total hip and knee } \\
\text { arthroplasty }\end{array}$ & $\begin{array}{l}\text { Modified frailty } \\
\text { index }\end{array}$ & CD 4 & Good \\
\hline Suskind & 95108 & $\begin{array}{l}\text { Multicenter cohort } \\
\text { study (NSQIP) }\end{array}$ & $\begin{array}{l}2007- \\
2013\end{array}$ & Prospective & $\begin{array}{l}\text { Common urological } \\
\text { surgery }\end{array}$ & $\begin{array}{l}\text { Modified frailty } \\
\text { index }\end{array}$ & $\begin{array}{l}\text { Major and } \\
\text { minor } \\
\text { complications }\end{array}$ & Good \\
\hline Suskind & 20794 & $\begin{array}{l}\text { Multicenter cohort } \\
\text { study (NSQIP) }\end{array}$ & $\begin{array}{l}2011- \\
2013\end{array}$ & Prospective & $\begin{array}{l}\text { Inpatient urological } \\
\text { surgery }\end{array}$ & $\begin{array}{l}\text { Modified frailty } \\
\text { index }\end{array}$ & Not reported & Good \\
\hline Tan & 83 & $\begin{array}{l}\text { Multicenter cohort } \\
\text { study }\end{array}$ & $\begin{array}{l}2008- \\
2010\end{array}$ & Prospective & Colorectal surgery & $\begin{array}{l}\text { Fried frailty } \\
\text { criteria }\end{array}$ & $\mathrm{CD} \geq 2$ & Fair \\
\hline Tegels & 127 & $\begin{array}{l}\text { Single-center } \\
\text { cohort study }\end{array}$ & $\begin{array}{l}2005- \\
2012\end{array}$ & Retrospective & $\begin{array}{l}\text { Surgery for gastric } \\
\text { cancer }\end{array}$ & $\begin{array}{l}\text { Groningen frailty } \\
\text { indicator }\end{array}$ & $\mathrm{CD} \geq 3$ & Fair \\
\hline Tsiouris & 1940 & $\begin{array}{l}\text { Multicenter cohort } \\
\text { study (NSQIP) }\end{array}$ & $\begin{array}{l}2005- \\
2010\end{array}$ & Prospective & Open lobectomy & $\begin{array}{l}\text { Modified frailty } \\
\text { index }\end{array}$ & CD 4 & Good \\
\hline Ugolini & 46 & $\begin{array}{l}\text { Single-center } \\
\text { cohort study }\end{array}$ & $\begin{array}{l}2009- \\
2012\end{array}$ & Prospective & $\begin{array}{l}\text { Elective colorectal cancer } \\
\text { surgery }\end{array}$ & $\begin{array}{l}\text { Groningen frailty } \\
\text { indicator; VES- } \\
13\end{array}$ & Not reported & Poor \\
\hline Uppal & 6551 & $\begin{array}{l}\text { Multicenter cohort } \\
\text { study (NSQIP) }\end{array}$ & $\begin{array}{l}2008- \\
2011\end{array}$ & Prospective & $\begin{array}{l}\text { Surgery for gynecologic } \\
\text { cancer }\end{array}$ & $\begin{array}{l}\text { Modified frailty } \\
\text { index }\end{array}$ & $\mathrm{CD} 4$ and 5 & Good \\
\hline
\end{tabular}

\section{Postoperative outcome predicted by frailty}

Table 1 shows the details of study demographics and methods of frailty measurement. In the selected studies, fifty-one were of prospective design and sample size ranged from 37 - 232352 patients. Gender distribution was reported in $93 \%$ of the studies with a proportion of females ranging from $0 \%$ in the study of Levy et al, describing a male population undergoing robot assisted radical prostatectomies, until $100 \%$ in the study of Courtney-Brooks et al, describing complications in elderly women undergoing gynecologic oncology surgery. Twenty-seven studies investigated the effect of frailty in oncological surgery (predominantly abdominal cancer surgery), four studies in vascular surgery, nine in orthopedic surgery, eleven in elective general surgery (predominantly intermediate - and high-risk surgery), four in emergency surgery and one study in transplant surgery. Thirty-one studies investigated the influence of frailty on 30-day mortality. Figure 2 shows a forest plot of this primary outcome with a pooled RR of 3.71 [95\% CI 2.894.77] (PI 1.38-9.97; I2=95\%) for frail patients compared to those who were not frail. The $95 \%$ prediction interval also showed exclusion of the null value.

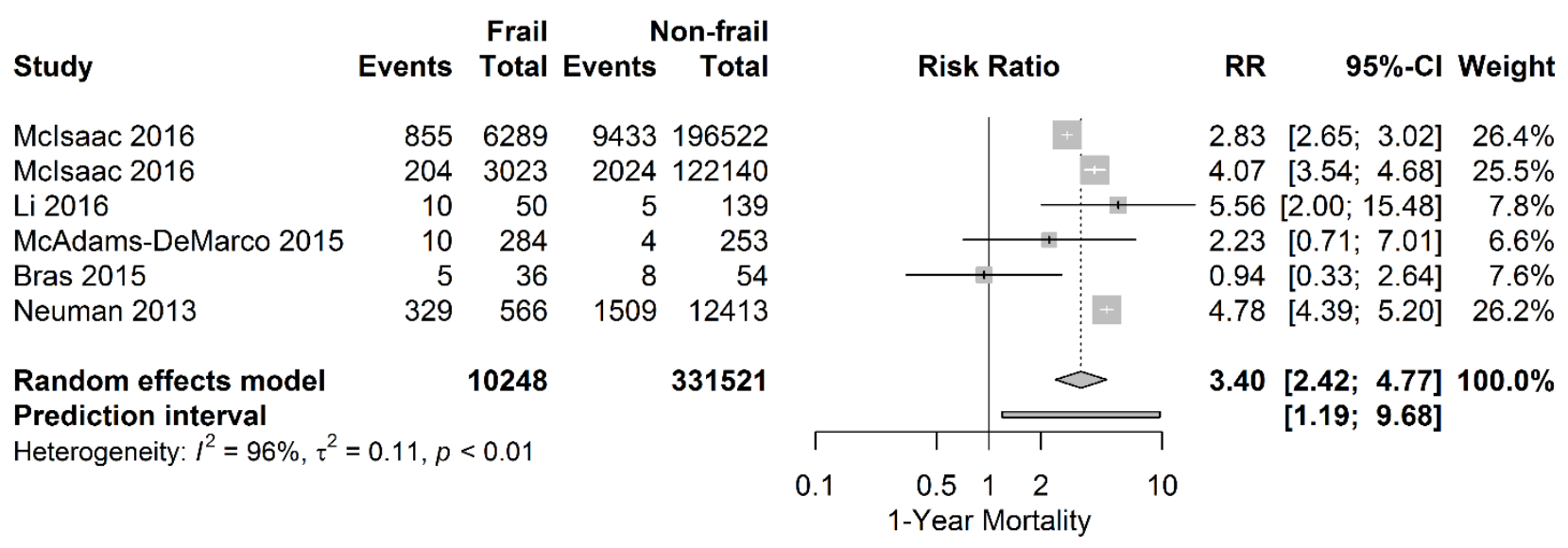

Figure 4. Forest plot 1-year mortality. The number of events (one-year mortality) and the total number of patients are depicted for frail and non-frail patients.

Stratified for frailty assessment tool, the association of frailty and 30-day mortality was observed according to the ACG frailty-defining diagnosis indicator, Fried frailty criteria, Frailty-based Risk Analysis Index and the Modified Frailty Index. 
Figure 3 shows the relationship between frailty and the occurrence of postoperative complications, stratified for frailty assessment tool. This adverse outcome was evaluated in 37 papers. Table 1 shows the predefined 30day complications reported by the authors, in most cases defined as suggested by the Clavien-Dindo classification system. Overall, a positive relationship between frailty and 30-day complications with a pooled RR of 2.39 [95\% CI 2.02-3.07] was observed (PI 0.96-5.69; I2=98\%), regardless of the frailty score used.

Stratified per surgical risk category, pooled RR's for 30-day mortality were 2.75 [95\% CI 2.48-3.05] for highrisk surgery (4 studies), RR 4.79 [95\% CI 3.42-6.70] for intermediate-risk surgery (18 studies) and RR 3.06 [95\% CI 2.35-3.97] for mixed surgical population (8 studies). The association of frailty and the primary outcome 30-day complications was also stratified per surgical risk category and again a positive relationship was observed with pooled RR's of 1.62 [95\% CI 1.43 -1.82] for high- risk surgery (3 studies) and RR 2.94 [95\% CI 2.44-3.54] for intermediate-risk surgery (24 studies).

Six studies investigated the association between frailty and one-year mortality (Fig. 4). In most of these studies, frailty increases the risk of one-year mortality with a pooled consequent risk ratio of 3.40 [95\% CI 2.424.77], (PI 1.19- 9.68; I2=96\%).

Figure 5 shows a forest plot, which summarizes the relationship between frailty and postoperative delirium. Four studies (438 patients) describe a positive relationship between frailty and POD with a pooled RR of 2.13 [95\% CI 1.23-3.67], (PI 0.64- 7.05; I2=0\%).

Figure 6 shows that frail patients seem to struggle to return to their own home, as these patients, described in ten studies (149 752 patients), have a twofold higher risk of being discharged to a specialized facility after surgery (RR 2.30 [95\% CI 1.81-2.92]), (PI 1.06- 4.96; I2=92\%). Just like in 30-day mortality and one-year mortality, the 95\% prediction interval for postoperative discharge location showed exclusion of the null value.

\begin{tabular}{|c|c|c|c|c|}
\hline Study & Events & $\begin{array}{l}\text { Frail } \\
\text { Total }\end{array}$ & $\begin{array}{l}\text { Non } \\
\text { Events }\end{array}$ & $\begin{array}{l}\text { n-frail } \\
\text { Total }\end{array}$ \\
\hline Kristjansson 2010 & 11 & 76 & 3 & 102 \\
\hline Partridge 2015 & 15 & 65 & 9 & 60 \\
\hline Leung 2011 & 13 & 35 & 3 & 18 \\
\hline Kua 2016 & 6 & 71 & 0 & 11 \\
\hline $\begin{array}{l}\text { Random effects model } \\
\text { Prediction interval } \\
\text { Heterogeneity: } I^{2}=0 \%, \tau^{2}\end{array}$ & $0, p=$ & 247 & & 191 \\
\hline
\end{tabular}

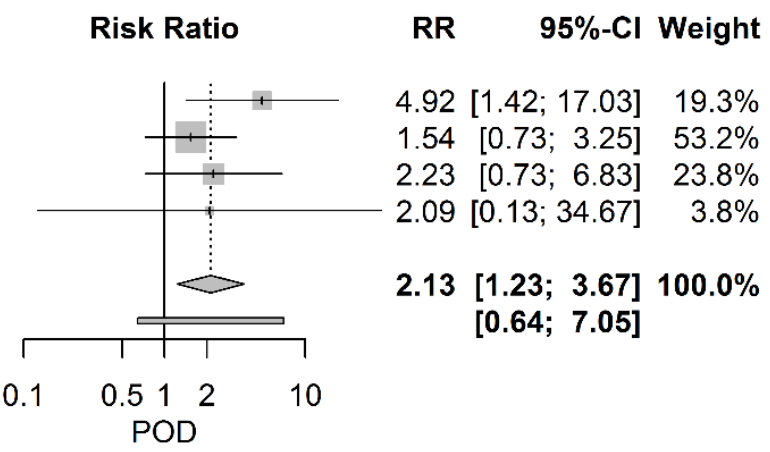

Figure 5. Forest plot postoperative delirium. The number of events (delirium) and the total number of patients are depicted for frail and non-frail patients.

A meta-regression analysis investigating showed no influence of age on primary outcome. Finally, to circumvent the issue of possible duplicate cases, the additional sensitivity analysis excluding studies using ACS-NSQIP database, showed an overall pooled RR of 3.62 [CI 95\% 2.21-5.92] (PI 1.46-8.98; I2=14\%) for 30day mortality

\section{DISCUSSION}

Since life expectancy keeps rising, the number of frail patients being offered for surgical treatment will dramatically increase. Frail patients are vulnerable and may excessively decompensate after stressors such as surgery, because of their lack of physiological reserve [13].
In this systematic review and meta-analysis, we found frailty to be a strong predictor of post surgical complications, delirium, institutionalization and all-cause mortality. After reviewing fifty-six articles, 30-day mortality shows the strongest association with preoperative frailty with almost 4 times increased risk.

Our results are congruent with several other reviews investigating the effect of frailty on postoperative outcome. [26-30] However, most of the previous studies focused on specific age groups, specific types of surgery, or specific frailty assessment tool. Therefore, extrapolations to a heterogeneous group of elderly and multimorbid patients should be limited.

The strength of the present study is the extensiveness of the search, the inclusion of different validated frailty 
scores and the inclusion of different types of non-cardiac surgery, both elective and acute. The quality of this metaanalysis is dependent on the quality of the studies reviewed. Of all studies included $95 \%$ were of at least fair quality, with more than half assessed as good quality. Ninety-one percent of all studies were prospectively designed.

\begin{tabular}{lrrrr} 
& & Frail & \multicolumn{2}{c}{ Non-frail } \\
Study & Events & Total Events & Total \\
& & & & \\
Mclsaac 2016 & 1708 & 3023 & 42260 & 122140 \\
Dasgupta 2009 & 10 & 16 & 34 & 109 \\
Krishnan 2014 & 94 & 122 & 11 & 56 \\
Cooper 2016 & 134 & 170 & 126 & 245 \\
Courtney-Brooks 2012 & 1 & 16 & 0 & 21 \\
Kim 2016 & 17 & 67 & 10 & 130 \\
Makary 2010 & 29 & 248 & 7 & 346 \\
Chimukangara 2016 & 14 & 43 & 83 & 842 \\
Suskind 2016 & 544 & 5554 & 596 & 16384 \\
Joseph 2016 & 48 & 82 & 55 & 138 \\
& & & & \\
Random effects model & & $\mathbf{9 3 4 1}$ & $\mathbf{1 4 0 4 1 1}$ \\
Prediction interval & & & & \\
Heterogeneity: $I^{2}=92 \%, \tau^{2}=0.10, p<0.01$ & &
\end{tabular}

Figure 6. Forest plot discharge to specialized facility. The number of events (discharge to a specialized facility) and the total number of patients are depicted for frail and non-frail patients.

The studies in this review and meta-analysis describe eleven different frailty assessment tools. Moreover, the surgical procedures included could basically be divided into six different groups, which will have contributed to the heterogeneity. Heterogeneity, as assessed with I2, t2, Cochran's Q and prediction intervals, was estimated as a high degree of statistical heterogeneity. Importantly, the association between frailty and outcome seems robust throughout the reviewed articles regardless of the frailty assessment tool used. Furthermore, prediction intervals of 30-day mortality, one-year mortality and postoperative discharge location showed exclusion of the null value, which strengthens our findings.

A plausible explanation may be the fact that frailty was consistently measured by instruments using physical, cognitive and functional domains. Studies using only measurements of body composition or patients' phenotype, such as sarcopenia, hypoalbuminemia or cachexia were not included, as these studies did not use an established frailty assessment tool. The frailty instrument used in most studies was the modified frailty index $(\mathrm{mFI})$, which has been validated as a reliable assessment tool in several studies [32-36]. It should be recommended that future studies focus on using a standardized, robust and validated frailty assessment tool,
Recently, relevant developments have been made towards methodological frameworks, in order to improve the reliability and applicability of prediction studies [31]. Although the authors found improved reporting standards in the last decade, poor reporting and poor methods are still a topic of concern and likely to limit the reliability in this type of clinical research.

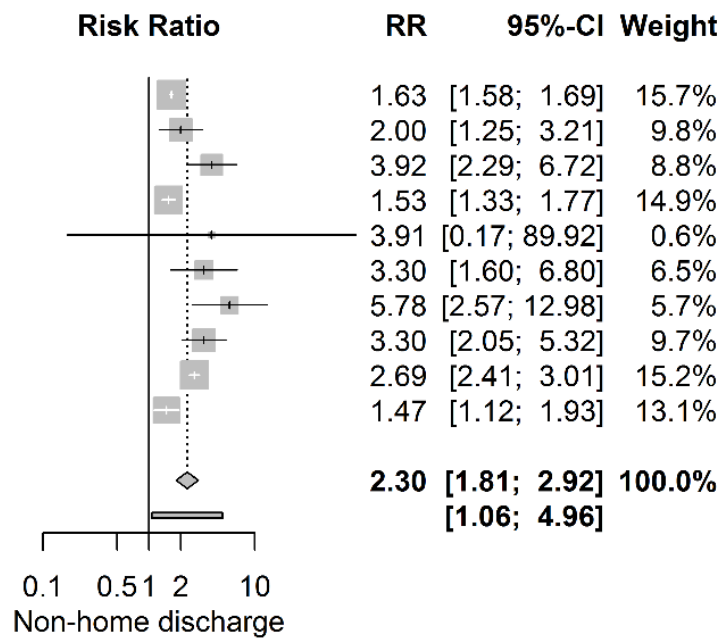

which is time-efficient and suitable for the medical staff to be conducted at patient's bedside.

Limitations of this study are those commonly seen with systematic reviews and meta-analysis. Hence, the results of this review and meta-analysis should be interpreted with caution. Besides the heterogeneity, another possible limitation is a variation among studies in the definition of discharge location. Despite these small differences, ten studies confirm that frail patients, when compared to healthier counterparts, struggle to return to their own home. Unfortunately, in many countries, availability of beds and nursing staff in specialized facilities are a topic of current concern. To overcome this limitation the need for rehabilitation or nursing home placement was defined as "not able to return home". Comparable heterogeneity was found within the definition of postoperative complications. Although most authors defined 30-day complications as suggested by the Clavien-Dindo classification system, others used the American College of Surgery National Surgical Quality Improvement Program definition, or other standardized complication definitions. It should be recommended that future studies in the area of frailty use a standardized postoperative complication definition as this might create a more accurate comparison. The International 
Consortium for Health Outcomes Measurement (ICHOM) recently developed the first global standard set of outcome measures in older persons. Their effort towards standardization of outcome measures can possibly improve care pathways and quality of care [37].

Although we have performed an exhaustive literature search, the broad scope of our research question could have resulted in the omission of some studies.

Many studies in this systematic review and metaanalysis are observational registry studies, but several studies have derived their outcomes from clinical trials. Since many studies have used the ACS NSQIP database, there may be studies, which are double counted from the same cohort of patients. However, table 1 shows that most of these studies observed different subgroups of patients, as well as different timeframes and kinds of surgical specialisms. Additionally, the sensitivity analysis we have performed, excluding studies using ACS-NSQIP database, demonstrated a positive relationship between frailty and primary outcomes. Finally, subgroup analyses gave insight in the heterogeneity among the types of surgery and different frailty assessment tools, but this stratification has the drawback of small groups.

In a previous study we have found that the occurrence of postoperative complications is an important prognostic factor of late mortality [38]. Efforts to improve postoperative outcome have predominantly focused on enhanced recovery protocols and the improvement of surgical and anesthetic techniques [39, 40]. The concept of prehabilitation is a modern and proactive approach, based on the principle that structured exercise over a period of weeks leads to a better cardiovascular, respiratory and muscular condition. Optimization of patients' functional capacity may provide a physiological buffer and enables the patient to better withstand the stress of surgery [39, 41, 42].

Preoperative identification of frail patients provides an opportunity for prehabilitation, which subsequently may lead to reduced postoperative morbidity. Besides prehabilitation, regionalization in health care might improve surgical outcome in complex oncological surgery. Regionalization is about enabling appropriate allocation and integration of health resources, focusing on the local populations needs. Frail patients may benefit from high-volume hospitals with high-volume surgeons in so called centers of excellence [43].

This study demonstrates that the presence of preoperative frailty increases the risk of adverse outcome after non-cardiac surgery. It should be noted that heterogeneity of the frailty scores is high, but associations with postoperative outcome are robust. Frailty status should be considered to be part of the preoperative screening, at least in patients who seem to have a lack of physiological reserve. Identification of potentially reversible health deficits is important, as may provide an opportunity to optimize patients' clinical condition prior to surgery. Conversely, irreversible frailty should be taken most seriously, as it can guide both clinician and patient in their decision making on whether the patient benefits from surgery or not.

\section{Acknowledgements}

Wichor M. Bramer, biomedical information specialist, Information Department University Library, Erasmus University Medical Center, Rotterdam.

\section{Supplementary Materials}

The Supplemenantry data can be found online at: www.aginganddisease.org/EN/10.14336/AD.2019.1024.

\section{References}

[1] Mangano DT (2004). Perioperative medicine: NHLBI working group deliberations and recommendations. J Cardiothorac Vasc Anesth, 18:1-6.

[2] Partridge JS, Harari D, Dhesi JK (2012). Frailty in the older surgical patient: a review. Age Ageing, 41:142147.

[3] Kristensen SD, Knuuti J, Saraste A, Anker S, Botker HE, De Hert S, et al. (2014). 2014 ESC/ESA Guidelines on non-cardiac surgery: cardiovascular assessment and management: The Joint Task Force on non-cardiac surgery: cardiovascular assessment and management of the European Society of Cardiology (ESC) and the European Society of Anaesthesiology (ESA). Eur J Anaesthesiol, 31:517-573.

[4] Lee DH, Buth KJ, Martin BJ, Yip AM, Hirsch GM (2010). Frail patients are at increased risk for mortality and prolonged institutional care after cardiac surgery. Circulation, 121:973-978.

[5] Polanczyk CA, Marcantonio E, Goldman L, Rohde LE, Orav J, Mangione CM, et al. (2001). Impact of age on perioperative complications and length of stay in patients undergoing noncardiac surgery. Ann Intern Med, 134:637-643.

[6] Browner WS, Li J, Mangano DT (1992). In-hospital and long-term mortality in male veterans following noncardiac surgery. The Study of Perioperative Ischemia Research Group. JAMA, 268:228-232.

[7] Arozullah AM, Khuri SF, Henderson WG, Daley J, Participants in the National Veterans Affairs Surgical Quality Improvement P (2001). Development and validation of a multifactorial risk index for predicting postoperative pneumonia after major noncardiac surgery. Ann Intern Med, 135:847-857.

[8] Huber CH, Goeber V, Berdat P, Carrel T, Eckstein F (2007). Benefits of cardiac surgery in octogenarians--a postoperative quality of life assessment. Eur J Cardiothorac Surg, 31:1099-1105. 
[9] Fruitman DS, MacDougall CE, Ross DB (1999). Cardiac surgery in octogenarians: can elderly patients benefit? Quality of life after cardiac surgery. Ann Thorac Surg, 68:2129-2135.

[10] Filsoufi F, Rahmanian PB, Castillo JG, Chikwe J, Silvay G, Adams DH (2007). Results and predictors of early and late outcomes of coronary artery bypass graft surgery in octogenarians. J Cardiothorac Vasc Anesth, 21:784-792.

[11] Baskett R, Buth K, Ghali W, Norris C, Maas T, Maitland A, et al. (2005). Outcomes in octogenarians undergoing coronary artery bypass grafting. CMAJ, 172:1183-1186.

[12] Sundermann S, Dademasch A, Rastan A, Praetorius J, Rodriguez H, Walther T, et al. (2011). One-year followup of patients undergoing elective cardiac surgery assessed with the Comprehensive Assessment of Frailty test and its simplified form. Interact Cardiovasc Thorac Surg, 13:119-123; discussion 123.

[13] Xue QL (2011). The frailty syndrome: definition and natural history. Clin Geriatr Med, 27:1-15.

[14] 2017. In Integrated Care for Older People: Guidelines on Community-Level Interventions to Manage Declines in Intrinsic Capacity. Geneva.

[15] Liberati A, Altman DG, Tetzlaff J, Mulrow C, Gotzsche PC, Ioannidis JP, et al. (2009). The PRISMA statement for reporting systematic reviews and meta-analyses of studies that evaluate healthcare interventions: explanation and elaboration. BMJ, 339:b2700.

[16] Dindo D, Demartines N, Clavien PA (2004). Classification of surgical complications: a new proposal with evaluation in a cohort of 6336 patients and results of a survey. Ann Surg, 240:205-213.

[17] De J, Wand AP (2015). Delirium Screening: A Systematic Review of Delirium Screening Tools in Hospitalized Patients. Gerontologist, 55:1079-1099.

[18] Kristensen SD, Knuuti J (2014). New ESC/ESA Guidelines on non-cardiac surgery: cardiovascular assessment and management. Eur Heart J, 35:2344-2345.

[19] Hayden JA, Cote P, Bombardier C (2006). Evaluation of the quality of prognosis studies in systematic reviews. Ann Intern Med, 144:427-437.

[20] Graham PL, Moran JL (2012). Robust meta-analytic conclusions mandate the provision of prediction intervals in meta-analysis summaries. J Clin Epidemiol, 65:503-510.

[21] IntHout J, Ioannidis JP, Rovers MM, Goeman JJ (2016). Plea for routinely presenting prediction intervals in metaanalysis. BMJ Open, 6:e010247.

[22] Velanovich V, Antoine H, Swartz A, Peters D, Rubinfeld I (2013). Accumulating deficits model of frailty and postoperative mortality and morbidity: Its application to a national database. J Surg Res, 183:104-110.

[23] Searle SD, Mitnitski A, Gahbauer EA, Gill TM, Rockwood K (2008). A standard procedure for creating a frailty index. BMC Geriatr, 8:24.

[24] Fuchshuber PR, Greif W, Tidwell CR, Klemm MS, Frydel C, Wali A, et al. (2012). The power of the National Surgical Quality Improvement Program-achieving a zero pneumonia rate in general surgery patients. Perm J, 16:39-45.
[25] Fried LP, Tangen CM, Walston J, Newman AB, Hirsch C, Gottdiener J, et al. (2001). Frailty in older adults: evidence for a phenotype. J Gerontol A Biol Sci Med Sci, 56:M146-156.

[26] Hewitt J, Long S, Carter B, Bach S, McCarthy K, Clegg A (2018). The prevalence of frailty and its association with clinical outcomes in general surgery: a systematic review and meta-analysis. Age Ageing, 47:793-800.

[27] Oakland K, Nadler R, Cresswell L, Jackson D, Coughlin PA (2016). Systematic review and meta-analysis of the association between frailty and outcome in surgical patients. Ann R Coll Surg Engl, 98:80-85.

[28] Lin HS, Watts JN, Peel NM, Hubbard RE (2016). Frailty and post-operative outcomes in older surgical patients: a systematic review. BMC Geriatr, 16:157.

[29] Panayi AC, Orkaby AR, Sakthivel D, Endo Y, Varon D, Roh D, et al. (2018). Impact of frailty on outcomes in surgical patients: A systematic review and meta-analysis. Am J Surg.

[30] Wang J, Zou Y, Zhao J, Schneider DB, Yang Y, Ma Y, et al. (2018). The Impact of Frailty on Outcomes of Elderly Patients After Major Vascular Surgery: A Systematic Review and Meta-analysis. Eur J Vasc Endovasc Surg, 56:591-602.

[31] Bouwmeester W, Zuithoff NP, Mallett S, Geerlings MI, Vergouwe Y, Steyerberg EW, et al. (2012). Reporting and methods in clinical prediction research: a systematic review. PLoS Med, 9:1-12.

[32] Ehlert BA, Najafian A, Orion KC, Malas MB, Black JH, Abularrage CJ (2016). Validation of a modified Frailty Index to predict mortality in vascular surgery patients. J Vasc Surg, 63:1595e1592-1601e1592.

[33] Abt NB, Richmon JD, Koch WM, Eisele DW, Agrawal $N$ (2016). Assessment of the predictive value of the modified frailty index for Clavien-Dindo grade IV critical care complications in major head and neck cancer operations. JAMA Otolaryngol Head Neck Surg, 142:658-664.

[34] Ali R, Schwalb JM, Nerenz DR, Antoine HJ, Rubinfeld I (2016). Use of the modified frailty index to predict 30day morbidity and mortality from spine surgery. $\mathbf{J}$ Neurosurg Spine, 25:537-541.

[35] Tsiouris A, Hammoud ZT, Velanovich V, Hodari A, Borgi J, Rubinfeld I (2013). A modified frailty index to assess morbidity and mortality after lobectomy. J Surg Res, 183:40-46.

[36] Uppal S, Igwe E, Rice LW, Spencer RJ, Rose SL (2015). Frailty index predicts severe complications in gynecologic oncology patients. Gynecol Oncol, 137:98101.

[37] Akpan A, Roberts C, Bandeen-Roche K, Batty B, Bausewein C, Bell D, et al. (2018). Standard set of health outcome measures for older persons. BMC Geriatr, 18:36.

[38] Tjeertes EK, Ultee KH, Stolker RJ, Verhagen HJ, Bastos Goncalves FM, Hoofwijk AG, et al. (2016). Perioperative Complications are Associated With Adverse Long-Term Prognosis and Affect the Cause of Death After General Surgery. World J Surg, 40:25812590 . 
[39] Wynter-Blyth V, Moorthy K (2017). Prehabilitation: preparing patients for surgery. BMJ, 358:j3702.

[40] Adamina M, Kehlet H, Tomlinson GA, Senagore AJ, Delaney CP (2011). Enhanced recovery pathways optimize health outcomes and resource utilization: a meta-analysis of randomized controlled trials in colorectal surgery. Surgery, 149:830-840.

[41] Moorthy K, Wynter-Blyth V (2017). Prehabilitation in perioperative care. Br J Surg, 104:802-803.

[42] Carli F, Scheede-Bergdahl C (2015). Prehabilitation to enhance perioperative care. Anesthesiol Clin, 33:17-33.

[43] Lumpkin S, Stitzenberg K (2018). Regionalization and Its Alternatives. Surg Oncol Clin N Am, 27:685-704.

[44] Krishnan M, Beck S, Havelock W, Eeles E, Hubbard RE, Johansen A (2014). Predicting outcome after hip fracture: Using a frailty index to integrate comprehensive geriatric assessment results. Age Ageing, 43:122-126.

[45] Cooper Z, Rogers SO, Ngo L, Guess J, Schmitt E, Jones RN, et al. (2016). Comparison of Frailty Measures as Predictors of Outcomes After Orthopedic Surgery. J Am Geriatr Soc, 64:2464-2471.

[46] Slaets JP (2006). Vulnerability in the elderly: frailty. Med Clin North Am, 90:593-601.

[47] Bras L, Peters TTA, Wedman J, Plaat BEC, Witjes MJH, van Leeuwen BL, et al. (2015). Predictive value of the Groningen Frailty Indicator for treatment outcomes in elderly patients after head and neck, or skin cancer surgery in a retrospective cohort. Clin Otolaryngol, 40:474-482.

[48] Huisman MG, Audisio RA, Ugolini G, Montroni I, Vigano A, Spiliotis J, et al. (2015). Screening for predictors of adverse outcome in onco-geriatric surgical patients: A multicenter prospective cohort study. Eur J Surg Oncol, 41:844-851.

[49] Reisinger KW, Van Vugt JLA, Tegels JJW, Snijders C, Hulsewé KWE, Hoofwijk AGM, et al. (2015). Functional compromise reflected by sarcopenia, frailty, and nutritional depletion predicts adverse postoperative outcome after colorectal cancer surgery. Ann Surg, 261:345-352.

[50] Tegels JJW, de Maat MFG, Hulsewé KWE, Hoofwijk AGM, Stoot JHMB (2014). Value of Geriatric Frailty and Nutritional Status Assessment in Predicting Postoperative Mortality in Gastric Cancer Surgery. J Gastrointest Surg, 18:439-446.

[51] Ugolini G, Pasini F, Ghignone F, Zattoni D, Reggiani MLB, Parlanti D, et al. (2015). How to select elderly colorectal cancer patients for surgery: a pilot study in an Italian academic medical center. Cancer Biol Med, 12:302-307.

[52] Kenig J, Zychiewicz B, Olszewska U, Barczynski M, Nowak W (2015). Six screening instruments for frailty in older patients qualified for emergency abdominal surgery. Arch Gerontol Geriatr, 61:437-442.

[53] Kua J, Ramason R, Rajamoney G, Chong MS (2016). Which frailty measure is a good predictor of early postoperative complications in elderly hip fracture patients? Arch Orthop Trauma Surg, 136:639-647.

[54] Li JL, Henderson MA, Revenig LM, Sweeney JF, Kooby DA, Maithel SK, et al. (2016). Frailty and one-year mortality in major intra-abdominal operations This study was presented at the World Congress of Endourology in London in October 2015. J Surg Res, 203:507.e501512.e501.

[55] Revenig LM, Canter DJ, Kim S, Liu Y, Sweeney JF, Sarmiento JM, et al. (2015). Report of a simplified frailty score predictive of short-term postoperative morbidity and mortality. J Am Coll Surg, 220:904-911.e901.

[56] Revenig LM, Canter DJ, Master VA, Maithel SK, Kooby DA, Pattaras JG, et al. (2014). A prospective study examining the association between preoperative frailty and postoperative complications in patients undergoing minimally invasive surgery. J Endourol, 28:476-480.

[57] Tan KY, Kawamura YJ, Tokomitsu A, Tang T (2012). Assessment for frailty is useful for predicting morbidity in elderly patients undergoing colorectal cancer resection whose comorbidities are already optimized. Am J Surg, 204:139-143.

[58] Kim S, Marsh AP, Rustowicz L, Roach C, Leng XI, Kritchevsky SB, et al. (2016). Self-reported mobility in older patients predicts early postoperative outcomes after elective noncardiac surgery. Anesthesiology, 124:815825.

[59] Leung JM, Tsai TL, Sands LP (2011). Preoperative frailty in older surgical patients is associated with early postoperative delirium. Anesth Analg, 112:1199-1201.

[60] Makary MA, Segev DL, Pronovost PJ, Syin D, BandeenRoche K, Patel P, et al. (2010). Frailty as a Predictor of Surgical Outcomes in Older Patients. J. Am. Coll. Surg., 210:901-908.

[61] McAdams-Demarco MA, Law A, King E, Orandi B, Salter M, Gupta N, et al. (2015). Frailty and mortality in kidney transplant recipients. Am J Transplant, 15:149154.

[62] Revenig LM, Canter DJ, Taylor MD, Tai C, Sweeney JF, Sarmiento JM, et al. (2013). Too frail for surgery? Initial results of a large multidisciplinary prospective study examining preoperative variables predictive of poor surgical outcomes. J Am Coll Surg, 217:665-670.e661.

[63] Courtney-Brooks M, Tellawi AR, Scalici J, Duska LR, Jazaeri AA, Modesitt SC, et al. (2012). Frailty: An outcome predictor for elderly gynecologic oncology patients. Gynecol Oncol, 126:20-24.

[64] Adams P, Ghanem T, Stachler R, Hall F, Velanovich V, Rubinfeld I (2013). Frailty as a predictor of morbidity and mortality in inpatient head and neck surgery. JAMA Otolaryngol Head Neck Surg, 139:783-789.

[65] Arya S, Kim SI, Duwayri Y, Brewster LP, Veeraswamy $\mathrm{R}$, Salam A, et al. (2015). Frailty increases the risk of 30day mortality, morbidity, and failure to rescue after elective abdominal aortic aneurysm repair independent of age and comorbidities. J Vasc Surg, 61:324-331.

[66] Augustin T, Burstein MD, Schneider EB, Morris-Stiff G, Wey J, Chalikonda S, et al. (2016). Frailty predicts risk of life-threatening complications and mortality after pancreatic resections. Surgery, 160:987-996.

[67] Brahmbhatt R, Brewster LP, Shafii S, Rajani RR, Veeraswamy R, Salam A, et al. (2016). Gender and frailty predict poor outcomes in infrainguinal vascular surgery. J Surg Res, 201:156-165. 
[68] Chappidi MR, Kates M, Patel HD, Tosoian JJ, Kaye DR, Sopko NA, et al. (2016). Frailty as a marker of adverse outcomes in patients with bladder cancer undergoing radical cystectomy. Urol Oncol Semin Orig Invest.

[69] Chimukangara M, Frelich MJ, Bosler ME, Rein LE, Szabo A, Gould JC (2016). The impact of frailty on outcomes of paraesophageal hernia repair. J Surg Res, 202:259-266.

[70] Cloney M, D'Amico R, Lebovic J, Nazarian M, Zacharia BE, Sisti MB, et al. (2016). Frailty in Geriatric Glioblastoma Patients: A Predictor of Operative Morbidity and Outcome. World Neurosurg, 89:362-367.

[71] Farhat JS, Velanovich V, Falvo AJ, Horst HM, Swartz A, Patton Jr JH, et al. (2012). Are the frail destined to fail? Frailty index as predictor of surgical morbidity and mortality in the elderly. J Trauma Acute Care Surg, 72:1526-1531.

[72] Flexman AM, Charest-Morin R, Stobart L, Street J, Ryerson CJ (2016). Frailty and postoperative outcomes in patients undergoing surgery for degenerative spine disease. Spine J, 16:1315-1323.

[73] Lascano D, Pak JS, Kates M, Finkelstein JB, Silva M, Hagen E, et al. (2015). Validation of a frailty index in patients undergoing curative surgery for urologic malignancy and comparison with other risk stratification tools. Urol Oncol Semin Orig Invest, 33:426.e421426.e412.

[74] Levy I, Finkelstein M, Bilal KH, Palese M (2017). Modified frailty index associated with Clavien-Dindo IV complications in robot-assisted radical prostatectomies: A retrospective study. Urol Oncol Semin Orig Invest.

[75] Louwers L, Schnickel G, Rubinfeld I (2016). Use of a simplified frailty index to predict Clavien 4 complications and mortality after hepatectomy: Analysis of the National Surgical Quality Improvement Project database. Am J Surg, 211:1071-1076.

[76] Mogal H, Vermilion SA, Dodson R, Hsu FC, Howerton R, Shen P, et al. (2017). Modified Frailty Index Predicts Morbidity and Mortality After Pancreaticoduodenectomy. Ann Surg Oncol:1-8.

[77] Mosquera C, Spaniolas K, Fitzgerald TL (2016). Impact of frailty on surgical outcomes: The right patient for the right procedure. Surgery, 160:272-280.

[78] Obeid NM, Azuh O, Reddy S, Webb S, Reickert C, Velanovich V, et al. (2012). Predictors of critical carerelated complications in colectomy patients using the National Surgical Quality Improvement Program: Exploring frailty and aggressive laparoscopic approaches. J Trauma Acute Care Surg, 72:878-883.

[79] Pearl JA, Patil D, Filson CP, Arya S, Alemozaffar M, Master VA, et al. (2017). Patient Frailty and Discharge Disposition Following Radical Cystectomy. Clin Genitourin Cancer.

[80] Phan K, Kim JS, Lee NJ, Somani S, Di Capua J, Kothari $\mathrm{P}$, et al. (2017). Frailty is associated with morbidity in adults undergoing elective anterior lumbar interbody fusion (ALIF) surgery. Spine J, 17:538-544.

[81] Suskind AM, Jin C, Cooperberg MR, Finlayson E, Boscardin WJ, Sen S, et al. (2016). Preoperative Frailty Is Associated With Discharge to Skilled or Assisted
Living Facilities After Urologic Procedures of Varying Complexity. Urology, 97:25-32.

[82] Suskind AM, Walter LC, Jin C, Boscardin J, Sen S, Cooperberg MR, et al. (2016). Impact of frailty on complications in patients undergoing common urological procedures: A study from the American College of Surgeons National Surgical Quality Improvement database. BJU Int.

[83] Shin JI, Keswani A, Lovy AJ, Moucha CS (2016). Simplified Frailty Index as a Predictor of Adverse Outcomes in Total Hip and Knee Arthroplasty. J Arthroplasty, 31:2389-2394.

[84] Shin JI, Kothari P, Phan K, Kim JS, Leven D, Lee NJ, et al. (2017). Frailty Index as a Predictor of Adverse Postoperative Outcomes in Patients Undergoing Cervical Spinal Fusion. Spine, 42:304-310.

[85] Rolfson DB, Majumdar SR, Tsuyuki RT, Tahir A, Rockwood K (2006). Validity and reliability of the Edmonton Frail Scale. Age Ageing, 35:526-529.

[86] Dasgupta M, Rolfson DB, Stolee P, Borrie MJ, Speechley M (2009). Frailty is associated with postoperative complications in older adults with medical problems. Arch Gerontol Geriatr, 48:78-83.

[87] Partridge JSL, Fuller M, Harari D, Taylor PR, Martin FC, Dhesi JK (2015). Frailty and poor functional status are common in arterial vascular surgical patients and affect postoperative outcomes. Int J Surg, 18:57-63.

[88] Rockwood K, Song X, MacKnight C, Bergman H, Hogan DB, McDowell I, et al. (2005). A global clinical measure of fitness and frailty in elderly people. CMAJ, 173:489-495.

[89] Hewitt J, Moug SJ, Middleton M, Chakrabarti M, Stechman MJ, McCarthy K (2015). Prevalence of frailty and its association with mortality in general surgery. Am J Surg, 209:254-259.

[90] Joseph B, Zangbar B, Pandit V, Fain M, Mohler MJ, Kulvatunyou N, et al. (2016). Emergency General Surgery in the Elderly: Too Old or Too Frail? Presented orally at the Surgical Forum of the American College of Surgeons 100th Annual Clinical Congress, San Francisco, CA, October 2014. J Am Coll Surg, 222:805813.

[91] Robinson TN, Wu DS, Pointer L, Dunn CL, Cleveland Jr JC, Moss M (2013). Simple frailty score predicts postoperative complications across surgical specialties. Am J Surg, 206:544-550.

[92] Saliba D, Elliott M, Rubenstein LZ, Solomon DH, Young RT, Kamberg CJ, et al. (2001). The Vulnerable Elders Survey: a tool for identifying vulnerable older people in the community. J Am Geriatr Soc, 49:16911699.

[93] Dale W, Hemmerich J, Kamm A, Posner MC, Matthews JB, Rothman R, et al. (2014). Geriatric assessment improves prediction of surgical outcomes in older adults undergoing pancreaticoduodenectomy: A prospective cohort study. Ann Surg, 259:960-965.

[94] Lieberman R AC, Weiner JP. 2003. Development and Evaluation of the Johns Hopkins University Risk Adjustment Models for Medicare ? Choice Plan Payment.: Baltimore, MD; Johns Hopkins University. 
[95] Neuman HB, Weiss JM, Leverson G, O'Connor ES, Greenblatt DY, Loconte NK, et al. (2013). Predictors of Short-Term Postoperative Survival after Elective Colectomy in Colon Cancer Patients $\geq 80$ Years of Age. Ann Surg Oncol, 20:1427-1435.

[96] McIsaac DI, Bryson GL, van Walraven C (2016). Association of Frailty and 1-Year Postoperative Mortality Following Major Elective Noncardiac Surgery: A Population-Based Cohort Study. JAMA Surg.

[97] McIsaac DI, Beaule PE, Bryson GL, Van Walraven C (2016). The impact of frailty on outcomes and healthcare resource usage after total joint arthroplasty: a populationbased cohort study. Bone Joint J, 98-B:799-805.

[98] Makary MA, Segev DL, Pronovost PJ, Syin D, BandeenRoche K, Patel P, et al. (2010). Frailty as a predictor of surgical outcomes in older patients. J Am Coll Surg, 210:901-908.

[99] Melin AA, Schmid KK, Lynch TG, Pipinos II, Kappes S, Longo GM, et al. (2015). Preoperative frailty risk analysis index to stratify patients undergoing carotid endarterectomy. J Vasc Surg, 61:683-689.

[100] Balducci L, Beghe C (2000). The application of the principles of geriatrics to the management of the older person with cancer. Crit Rev Oncol Hematol, 35:147154.

[101] Kristjansson SR, Nesbakken A, Jordhøy MS, Skovlund E, Audisio RA, Johannessen HO, et al. (2010). Comprehensive geriatric assessment can predict complications in elderly patients after elective surgery for colorectal cancer: A prospective observational cohort study. Crit Rev Oncol Hematol, 76:208-217.

[102] Lasithiotakis K, Petrakis J, Venianaki M, Georgiades G, Koutsomanolis D, Andreou A, et al. (2013). Frailty predicts outcome of elective laparoscopic cholecystectomy in geriatric patients. Surg Endosc Interv Tech, 27:1144-1150.

[103] Kim SW, Han HS, Jung HW, Kim KI, Hwang DW, Kang SB, et al. (2014). Multidimensional Frailty Score for the Prediction of Postoperative Mortality Risk. JAMA Surg., 149:633-640. 